\title{
Hyponatremia presenting with hourly fluctuating urine osmolality
}

\section{Raku Son'1, Masahiko Nagahama1', Fumiaki Tanemoto', Yugo Ito', Fumika Taki', Ryosuke Tsugitomi² and Masaaki Nakayama'}

'Department of Nephrology, St. Luke's International Hospital, Tokyo, Japan and 2Department of Pulmonary Medicine, Thoracic Center, St. Luke's International Hospital, Tokyo, Japan
Correspondence should be addressed to M Nagahama Email

managaha@luke.ac.jp

\section{Summary}

The etiology of hyponatremia is assessed based on urine osmolality and sodium. We herein describe a 35-year-old Asian man with pulmonary tuberculosis and perforated duodenal ulcer who presented with hyponatremia with hourly fluctuating urine osmolality ranging from 100 to $600 \mathrm{mosmol} / \mathrm{kg}$, which resembled urine osmolality observed in typical polydipsia and SIADH simultaneously. Further review revealed correlation of body temperature and urine osmolality. Since fever is a known non-osmotic stimulus of ADH secretion, we theorized that hyponatremia in this patient was due to transient ADH secretion due to fever. In our case, empiric exogenous glucocorticoid suppressed transient non-osmotic $\mathrm{ADH}$ secretion and urine osmolality showed highly variable concentrations. Transient ADH secretion-related hyponatremia may be underrecognized due to occasional empiric glucocorticoid administration in patients with critical illnesses. Repeatedly monitoring of urine chemistries and interpretation of urine chemistries with careful review of non-osmotic stimuli of ADH including fever is crucial in recognition of this etiology.

\section{Learning points:}

- Hourly fluctuations in urine osmolality can be observed in patients with fever, which is a non-osmotic stimulant of ADH secretion.

- Repeated monitoring of urine chemistries aids in the diagnosis of the etiology underlying hyponatremia, including fever, in patients with transient ADH secretion.

- Glucocorticoid administration suppresses ADH secretion and improves hyponatremia even in the absence of adrenal insufficiency; the etiology of hyponatremia should be determined carefully in these patients.

\section{Background}

Hyponatremia is the most common electrolyte disturbance observed in hospitalized patients and is associated with increased mortality (1); however, identifying the etiology of hyponatremia is complex. Clinical guidelines suggest a focus on urine chemistries (osmolality and sodium) rather than volume status assessment (2). The urine chemistry parameters are more objective and easier to interpret compared with the volume status and thus facilitate a more precise diagnosis of the hyponatremia etiology compared with the traditional volume status assessment. However, the diagnosis of hyponatremia remains challenging in patients with multifactorial etiologies.

We herein present a challenging case of hyponatremia in a patient with hourly fluctuating urine osmolality, which turned out to be caused by non-osmotic ADH secretion and empiric exogenous glucocorticoid administration. We also review the potential causes of highly variable urine osmolality and approaches in interpretation of this complicated presentation. 


\section{Case presentation}

A 35-year-old homeless Asian male with no significant medical history presented with 1 week of cough and hemoptysis and 1 day of right lower quadrant abdominal pain. He was admitted with the diagnoses of pulmonary tuberculosis and perforated duodenal ulcer. He underwent emergent surgery for perforation closure on the admission day. Postoperatively, he had persistent hypotension despite high-dose vasopressors, accompanied with hypoglycemia (blood glucose, 60 $\mathrm{mg} / \mathrm{dL}$ ) and hyponatremia (serum sodium, $128 \mathrm{mEq} / \mathrm{L}$ ). Hydrocortisone at $100 \mathrm{mg}$ three times daily was initiated for critical illness-related corticosteroid insufficiency, in addition to the standard tuberculosis treatment with rifampicin, isoniazid, pyrazinamide, and streptomycin. Additionally, he was administered acetaminophen as needed for spiking fever episodes due to either tuberculosis or possible drug allergy. Hydrocortisone was tapered over 5 days, and his serum sodium level, which was $140 \mathrm{mEq} / \mathrm{L}$ at the time of corticosteroid discontinuation, gradually decreased to $125 \mathrm{mEq} / \mathrm{L}$ over the next week. On day 13 of admission, he was referred to the nephrology department for consultation regarding the management of progressive hyponatremia.

At the time of the consultation, he denied any pain including headache, nausea, or vomiting. In addition to the anti-tuberculosis medications, he was on levofloxacin, meropenem, and famotidine. He was on total parenteral nutrition and supplementation for potassium, phosphate, and magnesium. Physical examination revealed that he was poorly nourished and had a nasogastric tube and tracheotomy. His vital signs were as follows: height, $165 \mathrm{~cm}$; weight, $44.6 \mathrm{~kg}$ (compared with the presumed body weight of $42.6 \mathrm{~kg}$ on admission); BMI, $16.5 \mathrm{~kg} / \mathrm{m}^{2}$; temperature, $37.9^{\circ} \mathrm{C}$; blood pressure, $106 / 61 \mathrm{mmHg}$ in supine position; heart rate, 110 beats/min; and oxygen saturation, $95 \%$ on ventilator $\left(\mathrm{FiO}_{2}, 0.25\right.$; positive end-expiratory pressure, $5 \mathrm{cmH}_{2} \mathrm{O}$; pressure support, 6 $\mathrm{cmH}_{2} \mathrm{O}$ ). He was alert and oriented, with a Glasgow Coma Scale score of E4TM6. He had moist mucous membranes, normal skin turgor, and slight edema on both upper extremities. The rest of the examination findings were unremarkable.

Laboratory studies at the time of admission and during the consultation are presented in Table 1. Whole trunk CT scan with contrast on admission revealed pulmonary infection and abdominal abscess. No calcification or enlargement of the adrenal glands was noted.
Table 1 Laboratory studies on admission and consultation.

\begin{tabular}{|c|c|c|}
\hline Parameter & $\begin{array}{c}\text { Value on } \\
\text { admission (day 1) }\end{array}$ & $\begin{array}{c}\text { Value on } \\
\text { consultation (day 13) }\end{array}$ \\
\hline \multicolumn{3}{|l|}{ Blood chemistries } \\
\hline Sodium (mEq/L) & 125 & 125 \\
\hline Potassium (mEq/L) & 4.5 & 3.7 \\
\hline Chloride (mEq/L) & 88 & 91 \\
\hline $\begin{array}{l}\text { Serum urea } \\
\text { nitrogen }(\mathrm{mg} / \mathrm{dL})\end{array}$ & 10.2 & 5.7 \\
\hline Creatinine (mg/dL) & 0.50 & 0.23 \\
\hline Glucose (mg/dL) & 68 & 105 \\
\hline $\begin{array}{l}\text { Serum osmolality } \\
\text { (mosmol/L) }\end{array}$ & - & 249 \\
\hline Calcium (mg/dL) & 7.3 & 6.4 \\
\hline Magnesium (mg/dL) & 1.7 & 1.9 \\
\hline Phosphorus (mg/dL) & 3.9 & 1.6 \\
\hline Albumin (g/dL) & 1.3 & 1.3 \\
\hline Uric acid (mg/dL) & - & 1.7 \\
\hline CRP (mg/dL) & 16.70 & 13.28 \\
\hline \multicolumn{3}{|l|}{ Atrial blood gases } \\
\hline $\mathrm{pH}$ & 7.262 & 7.463 \\
\hline $\mathrm{PCO}_{2}(\mathrm{mmHg})$ & 53.4 & 37.6 \\
\hline $\mathrm{PO}_{2}(\mathrm{mmHg})$ & $171.0\left(\mathrm{FiO}_{2}: 0.6\right)$ & $69.7\left(\mathrm{FiO}_{2}: 0.25\right)$ \\
\hline Bicarbonate (mEq/L) & 23.4 & 26.5 \\
\hline \multicolumn{3}{|l|}{ Complete blood count } \\
\hline Hemoglobin (g/dL) & 12.8 & 7.5 \\
\hline Hematocrit (\%) & 38.2 & 21.8 \\
\hline WBC count $\left(\times 10^{3} / \mu \mathrm{L}\right)$ & 15.2 & 13.0 \\
\hline Platelets $\left(\times 10^{3} / \mu \mathrm{L}\right)$ & 171 & 199 \\
\hline \multicolumn{3}{|l|}{ Urinary chemistries } \\
\hline Sodium (mEq/L) & - & 169 \\
\hline Potassium (mEq/L) & - & 34 \\
\hline $\begin{array}{l}\text { Urine urea nitrogen } \\
(\mathrm{mg} / \mathrm{dL})\end{array}$ & - & 297 \\
\hline Creatinine (mg/dL) & - & 35 \\
\hline $\begin{array}{l}\text { Osmolality } \\
\text { (mosmol/L) }\end{array}$ & - & 529 \\
\hline $\mathrm{FE}_{\mathrm{Na}}(\%)$ & - & 0.9 \\
\hline $\mathrm{FE}_{\mathrm{UA}}(\%)$ & - & 12.9 \\
\hline $\mathrm{FE}_{\mathrm{p}}(\%)$ & - & 12.1 \\
\hline
\end{tabular}

Conversion factors for units: serum and urine urea nitrogen from $\mathrm{mg} / \mathrm{dL}$ to $\mathrm{mmol} / \mathrm{L}, 0.357$; calcium from $\mathrm{mg} / \mathrm{dL}$ to $\mathrm{mmol} / \mathrm{L}, 0.2495$; phosphorus from $\mathrm{mg} / \mathrm{dL}$ to $\mathrm{mmol} / \mathrm{L}, 0.3229$.

CRP, C-reactive protein; $\mathrm{FE}_{\mathrm{Na}}$, fractional excretion of sodium; $\mathrm{FE}_{\mathrm{p} \text {, }}$ fractional excretion of phosphate; $\mathrm{FE}_{\mathrm{UA}}$, fractional excretion of uric acid; $\mathrm{PCO}_{2}$, partial pressure of carbon dioxide; $\mathrm{PMN}$, polymorphonuclear; $\mathrm{PO}_{2}$, partial pressure of oxygen; WBC, white blood cell.

\section{Investigation}

The adrenocorticotropic hormone (ACTH) stimulation test using i.v. tetracosactide acetate $(250 \mu \mathrm{g})$ on admission day 9 after hydrocortisone discontinuation ruled out adrenal insufficiency, with peak cortisol value of $28.17 \mu \mathrm{g} / \mathrm{dL}$ (3). The thyroid function tests were not significant. The levels of plasma renin activity, serum aldosterone, $\alpha$-human atrial natriuretic polypeptide, 
and amino-terminal pro-brain natriuretic peptide were $5.6 \mathrm{ng} / \mathrm{mL} / \mathrm{h}, 61 \mathrm{pg} / \mathrm{mL}, 28.7 \mathrm{pg} / \mathrm{mL}$, and $457.4 \mathrm{pg} / \mathrm{mL}$, respectively. His antidiuresis hormone (ADH) was elevated at $1.7 \mathrm{pg} / \mathrm{mL}$ despite hyponatremia. Further calculations revealed that the patient had been administered 3000 $\mathrm{mL}$ fluid per day, including total parental nutrition and antibiotics for several days, which comprised $97 \mathrm{mEq} / \mathrm{L}$ sodium and $13 \mathrm{mEq} / \mathrm{L}$ potassium. Based on the volume status assessment and the repeatedly high urine osmolality and urine sodium levels, the patient was diagnosed with the syndrome of inappropriate antidiuresis hormone (SIADH).

\section{Treatment}

Since water restriction and increased solute intake would change the composition of total parental nutrition, furosemide (5 mg intravenously) was chosen as the initial SIADH treatment, which failed to dilute the urine osmolality. Although the patient was asymptomatic, his sodium level dropped to $124 \mathrm{mEq} / \mathrm{L}$ overnight after the consultation. Tolvaptan $(7.5 \mathrm{mg})$ was administered for progressive hyponatremia; however, the urine chemistries revealed an unexpectedly diluted urine before the tolvaptan administration with no apparent cause, accompanied by changes in urine sodium level and urine osmolality from 190 to $20 \mathrm{mEq} / \mathrm{L}$ and from 515 to $155 \mathrm{mosmol} / \mathrm{L}$, respectively. The urine sodium level was increased rapidly with a large volume of urine output (1200 $\mathrm{mL}$ in $2 \mathrm{~h}$ ). The overcorrection of the hyponatremia was prevented by 75 $\mu \mathrm{g}$ desmopressin administered via a nasal spray. The urine osmolality was fluctuating hourly between 100 and 600 mosmol $/ \mathrm{kg}$, resembling the alternating urine osmolality observed in typical polydipsia and SIADH (Fig. 1).

\section{Outcome and follow-up}

The patient did not complain of any symptoms; therefore, no further intervention was sought. His serum sodium level gradually returned to $135 \mathrm{mEq} / \mathrm{L}$ over the next 2 weeks. However, 40 days after the initial admission, his respiratory state deteriorated. He was transferred to the intensive care unit, and his serum sodium level dropped to $129 \mathrm{mEq} / \mathrm{L}$ again at the time of transfer (Fig. 1). The second ACTH stimulation test suggested possible adrenal insufficiency, with peak cortisol value of $16.47 \mu \mathrm{g} / \mathrm{dL}$ (3). Hydrocortisone (200 mg daily), empirically initiated by the intensive care unit team, gradually corrected the serum sodium level to $140 \mathrm{mEq} / \mathrm{L}$. Hydrocortisone was tapered off, and he was discharged with a serum sodium level of

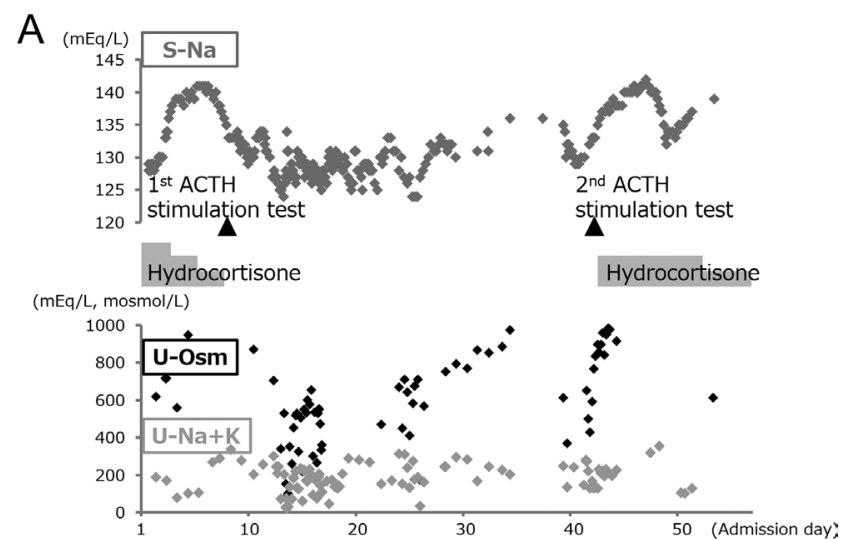

B

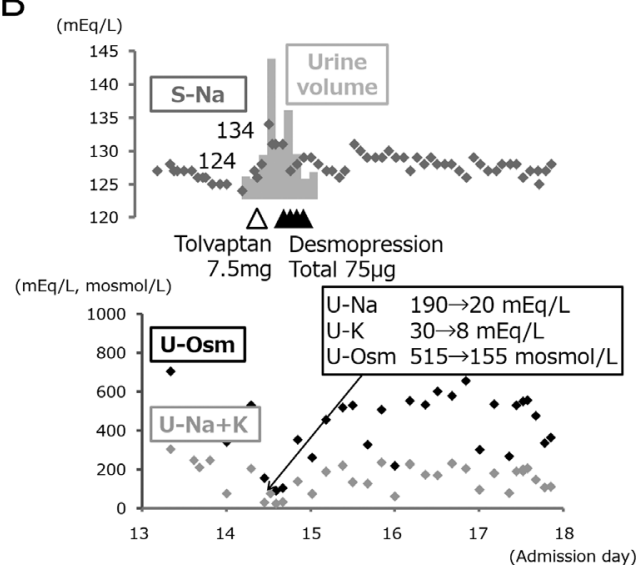

Figure 1

Clinical course of the patient including serum sodium levels, urine osmolality, and the sum of urine sodium and potassium levels $(A)$ through the entire presentation and $(B)$ during the 2 weeks after the consultation.

$138 \mathrm{mEq} / \mathrm{L}$ and a randomly measured cortisol level of 8.49 $\mu \mathrm{g} / \mathrm{dL}$ without corticosteroid supplementation.

\section{Discussion}

Hyponatremia is common in patients with tuberculosis, with prevalence rates reaching $62 \%$ (4). The causes of tuberculosis-related hyponatremia include SIADH directly due to infection or anti-tuberculosis medications and adrenal insufficiency related to disseminated adrenal tuberculosis (5). In the current patient, the urine osmolality and urine sodium levels were repeatedly high at the time of consultation. In addition to the urine chemistries, his euvolemic presentation and the normal ACTH stimulation test results initially supported the diagnosis of SIADH. However, his hyponatremia was overcorrected with an unexpected drop in urine osmolality to $150 \mathrm{mosmol} / \mathrm{L}$. The erratic, hourly changes in urine osmolality could not be explained simply as a typical SIADH presentation. 
Table 2 Causes of hyponatremia associated with unintentional overcorrection (7).

\section{Causes of hyponatremia}

Hypovolemia

Beer potomania, tea and toast diet

Thiazide diuretics

SSRIS

Desmopressin

Hypopituitarism

Addison disease

Hypoxemia

Nausea, surgery, pain, or stress

\section{Mechanism of escape from antidiuresis}

Volume repletion reverses baroreceptor-mediated vasopressin secretion Increased solute intake enhances delivery of glomerular filtrate to distal diluting sites Discontinuation of diuretic restores diluting function of the distal tubule Discontinuation of antidepressant eliminates drug-induced SIADH Discontinuation of synthetic vasopressin eliminates antidiuretic state Cortisol replacement restores ability to suppress vasopressin secretion Volume and cortisol replacement

Correction of hypoxemia eliminates non-osmotic stimulus for vasopressin Spontaneous resolution of SIADH

SIADH, syndrome of inappropriate secretion of antidiuretic hormone; SSRI, selective serotonin reuptake inhibitor.

The clinical course of the changes in urine osmolality and serum sodium levels are shown in Fig. 1. In the current patient, type of the administered i.v. fluids, respirator settings, and medications were constant, whereas the urine osmolality fluctuated significantly. Erratic, unregulated $\mathrm{ADH}$ secretion is commonly observed in SIADH (6); up to $30 \%$ of the patients present with wide ADH fluctuations, accompanied with changes in urine osmolality (6). However, urine osmolality is usually not suppressed to the extent of maximum antidiuresis (6), which rendered the SIADH diagnosis less likely in the current patient. Since no clear relationship between urine osmolality and serum sodium was observed, the causes of non-osmotic ADH secretion were investigated. Non-osmotic ADH secretion is clinically important because unintentional hyponatremia overcorrection might occur following the resolution of the etiology (7) (Table 2). None of the causes summarized in Table 2 was observed in the current patient, which raised the possibility of spiking fever episodes as the cause of erratic $\mathrm{ADH}$ secretion.

The physiologic association between fever and $\mathrm{ADH}$ secretion has been well recognized in humans as well as in animal models since 1960s. Although the exact mechanism is unknown, interleukin $1-\beta$ and tumor necrosis factor- $\alpha$ are suggested to cause the ADH secretion (8). The current patient experienced spiking fever episodes due to either tuberculosis or drug allergy during the week after the consultation, and there was a close correlation between his body temperature and urine osmolality (Fig. 2). We therefore speculated that non-osmotic $\mathrm{ADH}$ secretion was caused by the spiking fever episodes, which were resolved by acetaminophen. Thus, the large fluctuations in the urine osmolality were attributed to the changes in body temperature and antipyretic use. The progressive hyponatremia following the spiking fever episodes was likely augmented due to the relatively hypotonic total parenteral nutrition. In contrast, the urine chemistries during the afebrile periods mimicked polydipsia.

Retrospectively, the improvement in hyponatremia by hydrocortisone at the time of initial admission and on day 40 after admission in the current patient raises the possibility of adrenal insufficiency. Additionally, his hyponatremia responded well to hydrocortisone. However, since exogeneous glucocorticoids suppress ADH secretion, hydrocortisone can improve hyponatremia regardless of the adrenal function (9). It is possible that the fever-induced $\mathrm{ADH}$ secretion in the current patient was suppressed by exogeneous glucocorticoids, resulting in the improvement of hyponatremia. Although it remains possible that the patient suffered relative adrenal insufficiency concurrently, the previously mentioned pathophysiology should be considered in addition to any abnormalities in adrenal function in patients with hourly fluctuating urine osmolality. Albeit still controversial, glucocorticoid administration in patients with fever and possible relative adrenal insufficiency is

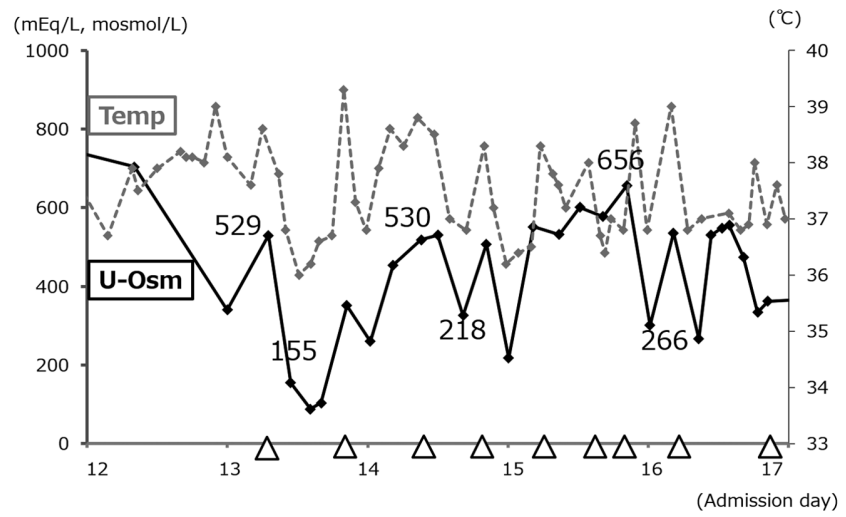

Figure 2

Clinical course of the patient's urine osmolality and body temperature within the first week following the consultation. The open triangles refer to the administration of acetaminophen. 
practiced commonly in intensive care units (10). Since the diagnosis of critical illness-related corticosteroid insufficiency is sometimes based only on empiric glucocorticoid administration, transient ADH secretion and hyponatremia can be improved by glucocorticoids even in patients without underlying adrenal insufficiency. It remains possible that the etiology of transient $\mathrm{ADH}$ secretion and hyponatremia seen in the current patient is underrecognized.

In conclusion, we herein presented a patient with hyponatremia and highly variable urine osmolality. Although SIADH contributed to hyperosmolar urine in the current case, highly variable urine osmolality seems to be related to fever-induced ADH secretion. The spiking fever episodes were associated with non-osmotic induction of $\mathrm{ADH}$ secretion, which resulted in the SIADH-like presentation, whereas the ADH secretion was reduced with the fever control, resulting in the polydipsia-like presentation. It is reasonable to assume that exogenous corticosteroids suppressed transient non-osmotic ADH secretion rather than correcting the underlying adrenal insufficiency in the current case. This case highlights the importance of repeated monitoring for urine chemistries and consideration of the causes of potential, transient non-osmotic induction of ADH secretion, including spiking fever.

\section{Declaration of interest}

The authors declare that there is no conflict of interest that could be perceived as prejudicing the impartiality of the research reported.

\section{Funding}

This research did not receive any specific grant from any funding agency in the public, commercial, or not-for-profit sector.

\section{Patient consent}

The patient provided written informed consent for the publication of all data and images of this case report.

\section{Author contribution statement}

All authors included in this article made substantial contributions to the analysis of the data included, as well as assisted with critical revisions of the writing, and approved the final version for submission for publication. R S, M N (Masahiko Nagahama), and R T participated to the endocrine assessment and treatment of the patient.

\section{References}

1 Chawla A, Sterns RH, Nigwekar SU \& Cappuccio JD. Mortality and serum sodium: do patients die from or with hyponatremia? Clinical Journal of the American Society of Nephrology 20116 960-965. (https:// doi.org/10.2215/CJN.10101110)

2 Spasovski G, Vanholder R, Allolio B, Annane D, Ball S, Bichet D, Decaux G, Fenske W, Hoorn EJ, Ichai C, et al. Clinical practice guideline on diagnosis and treatment of hyponatraemia. European Journal of Endocrinology 2014170 G1-G47. (https://doi.org/10.1530/ EJE-13-1020)

3 Ospina NS, Nofal AAl, Bancos I, Javed A, Benkhadra K, Kapoor E, Lteif AN, Natt N \& Murad MH. ACTH stimulation tests for the diagnosis of adrenal insufficiency: systematic review and metaanalysis. Journal of Clinical Endocrinology and Metabolism 2016101 427-434. (https://doi.org/10.1210/jc.2015-1700)

4 Bokam BR \& Badikillaya VU. Prevalence of hyponatremia in pulmonary tuberculosis - a pilot study from a tertiary care center in South India. International Journal of Medical Science and Public Health 20176 75-79. (https://doi.org/10.5455/ijmsph.2017.20062016560)

5 Vinnard C \& Blumberg EA. Endocrine and metabolic aspects of tuberculosis. Microbiology Spectrum 20175 163-178. (https://doi. org/10.1128/microbiolspec.TNMI7-0035-2016)

6 Robertson GL. Regulation of arginine vasopressin in the syndrome of inappropriate antidiuresis. American Journal of Medicine 2006119 (Supplement 1) S36-S42. (https://doi.org/10.1016/j. amjmed.2006.05.006)

7 Sterns RH, Hix JK \& Silver S. Treating profound hyponatremia: a strategy for controlled correction. American Journal of Kidney Diseases 201056 774-779. (https://doi.org/10.1053/j.ajkd.2010.04.020)

8 Takamata A, Mack GW, Stachenfeld NS \& Nadel ER. Body temperature modification of osmotically induced vasopressin secretion and thirst in humans. American Journal of Physiology: Regulatory, Integrative and Comparative Physiology 2017269 R874-R880.

9 Bähr V, Franzen N, Oelkers W, Pfeiffer AFH \& Diederich S. Effect of exogenous glucocorticoid on osmotically stimulated antidiuretic hormone secretion and on water reabsorption in man. European Journal of Endocrinology 2006155 845-848. (https://doi.org/10.1530/ eje.1.02299)

10 Annane D, Pastores SM, Arlt W, Balk RA, Beishuizen A, Briegel J, Carcillo J, Christ-Crain M, Cooper MS, Marik PE, et al. Critical IllnessRelated Corticosteroid Insufficiency (CIRCI): a narrative review from a multispecialty task force of the Society of Critical Care Medicine (SCCM) and the European Society of Intensive Care Medicine (ESICM). Critical Care Medicine 201745 2089-2098. (https://doi. org/10.1097/CCM.0000000000002724)

Received in final form 10 March 2020

Accepted 21 April 2020 